\title{
Studying the Antibody Repertoire after Vaccination: Practical Applications
}

\author{
Jacob D. Galson ${ }^{1}$, Andrew J. Pollard ${ }^{1}$, Johannes Trück ${ }^{1}$ \& Dominic F. Kelly ${ }^{1}$ \\ ${ }^{1}$ Oxford Vaccine Group, Department of Paediatrics, University of Oxford and the NIHR Oxford Biomedical Research \\ Centre, Oxford, UK
}

\begin{abstract}
Nearly all licensed vaccines have been developed to confer protection against infectious diseases by stimulating the production of antibodies by B cells, but the nature of a successful antibody response has been difficult to capture. Recent advances in next-generation sequencing technology have allowed high-resolution characterization of the antibody repertoire, and of the changes that occur following vaccination. These approaches have yielded important insights into the $B$ cell response, and have raised the possibility of using specific antibody sequences as measures of vaccine immunogenicity. Here, we review recent findings based on antibody repertoire sequencing, and discuss potential applications of these new technologies and of the analyses of the increasing volume of antibody sequence data in the context of vaccine development.
\end{abstract}

\footnotetext{
Corresponding authors: Galson, J.D. (jacob.galson@ paediatrics.ox.ac.uk)

Pollard, A.J. (andrew.pollard@paediatrics.ox.ac.uk)
}

Keywords: antibody repertoire; B cell receptor repertoire; VDJ; vaccination 


\section{Introduction}

Since the development of the first vaccine by Edward Jenner in 1796, vaccination has become one of the most important public health interventions worldwide. Where effective vaccines are used, they have greatly reduced the burden of infectious disease, preventing millions of deaths each year [1]. Most licensed vaccines confer protection against disease by stimulating B cells to produce pathogen-specific antibodies. The number of circulating B cell clones with distinct antibody heavy chain rearrangements is estimated to be at least $10^{6}$ [2-4], although there is the theoretical potential for every one of a human adult's $10^{11} \mathrm{~B}$ cells to produce a unique antibody variant [5]; such diversity is a key element in the recognition of any potential pathogen. The sum of all circulating antibodies is known as the antibody repertoire.

Antibodies are large molecules, consisting of paired heavy and light polypeptide chains. These form a variable $(\mathrm{V})$ antigen-binding region (known as $\mathrm{V}_{\mathrm{H}}$ and $\mathrm{V}_{\mathrm{L}}$ for the heavy and light chains respectively), as well as a constant (C) region [6]. Variability is concentrated in the three complementarity-determining regions (CDR1-3), which form a variety of tertiary structures to bind different antigens [7]. Initial $\mathrm{V}_{\mathrm{H} / \mathrm{L}}$ diversity is generated during B cell development by somatic recombination of multiple variable (V), diversity (D; $V_{H}$ only) and joining $(J)$ gene segments, to form functional $V_{H}$ and $V_{L}$ regions [8]. Diversity is increased at the junctions between segments by the addition of palindromic (P) and nontemplated (N) nucleotides, and exonuclease activity leading to potential nucleotide deletion. During response to an antigen, further $\mathrm{V}_{\mathrm{H} / \mathrm{L}}$ diversification occurs through rounds of somatic hypermutation, followed by selection of B cells for improved antigen binding in the germinal center (GC) [9].

An individual's antibody repertoire may be determined by a variety of factors including their genotype and chromatin structure [10,11], antigen exposure history [12] and age [12-17]. The diversity of the antibody repertoire makes it difficult to study, but improvements in next-generation sequencing (NGS) over the last decade now allow parallel sequencing of millions of antibody sequences (Box 1), making indepth studies of the repertoire possible. There is an increasing body of data characterizing changes in the antibody repertoire following vaccination (influenza [4,13,16,18-21], tetanus [18,22,23], Haemophilus influenzae type b $[24,25]$ and Streptococcus pneumoniae $[13,26]$ ), and natural infection (including influenza 
[27-32], rotavirus [33-36], HIV [37-44], hepatitis C [45], cytomegalovirus (CMV) [12], Epstein-Barr virus (EBV) [12], Staphylococcus aureus [46] and dengue [47-51] among others). The majority of the vaccine studies are based on low-resolution methods for characterizing the repertoire, but there are six recent studies, that use NGS for in-depth characterization (Table 1).

Despite the increasing number of studies of the antibody repertoire, there is little mention of the application of these data, with most publications instead focusing on methods development $[18,52]$. Here, we summarize recent advances in repertoire sequencing, and the insight this has given into the antibody repertoire. We discuss both the use of vaccines as a tool with which to investigate the antibody repertoire, and also the use of antibody repertoire sequencing as a tool for vaccinology that can potentially be applied to aid the development and assessment of new vaccines and vaccine schedules.

\section{Methods for studying the Antibody Repertoire}

\section{Low-resolution methods}

The earliest studies of the antibody repertoire used isoelectric focusing of antibodies on polyacrylamide gels [53], resolving the antibodies into patterns of discrete bands based on their isoelectric pH-values. CDR3 size spectratyping, a polymerase chain reaction (PCR) and electrophoresis-based method for determining CDR3 diversity based on nucleotide length distribution, can also give some insight into the antibody repertoire after vaccination [14]. The advent of Sanger sequencing allowed the exact nucleotide sequences coding for specific antibodies to be determined, albeit in small numbers [54]. Lymphocytes can be isolated after vaccination, followed by production of immortalized cell lines. Rearranged immunoglobulin DNA can then be amplified from these cell lines, and sequenced [24,55]. Additionally, cloning amplified DNA into expression vectors allows functional characterization of sequences for antigen-specificity [56]. Fluorescenceactivated single-cell sorting (FACS) has allowed more precise definition of the B cell subsets being studied. Sufficient DNA for sequencing or cloning can be generated from single cells by clonal expansion using culture [33], or single-cell reverse transcription PCR [20,57-59]. 
Such methods have been used to investigate small numbers of monoclonal antibodies (mAbs) generated against a variety of antigens, and although limited by cell numbers, we now know some antigenspecific sequences used in response to vaccines against influenza, tetanus, Haemophilus influenzae type b (Hib) and some serotypes of Streptococcus pneumoniae (Table 1), as well as natural infection (reviewed in [56]). These studies have shown that the B cell response to an antigen with a simple biochemical structure, such as Hib polysaccharide, appears markedly oligoclonal, with similar clones (predominantly $\mathrm{V}_{\mathrm{H}} 3-23$ [24,55,60,61], with a conserved 'GYGMD' CDR3 amino acid motif) dominating the repertoire in different individuals (although it should be noted that these studies were limited to a total of 19 individuals, and 25 sequences between them) [62]. Repertoire diversity is also restricted for protein antigens (e.g. tetanus toxoid, influenza haemagglutinin (HA)) [20,22,23], but the expanded clones differ more between individuals (again limited to at most 14 individuals, and $<500$ sequences per individual in any study) [20,22]. Even in a single individual, there appears to be little similarity in the response after repeated tetanus toxoid vaccination, with only one third of the clones sequenced being shared between vaccination events [22].

\section{NGS methods}

The advent of NGS (Box 1) enabled the sequencing of antibody genes from millions of cells simultaneously, giving greater insight into the entire antibody repertoire. An in-depth antibody repertoire study was initially conducted in zebrafish; this is a useful model system due to its small number of antibody-producing B cells (approximately 300,000 ), which is 5 orders of magnitude lower than in humans [63]. This study gave the first insights into the entire antibody repertoire of an organism, showing that zebrafish use 50-86\% of all possible VDJ combinations, and the VDJ frequency distribution is similar between individual zebrafish. It is not possible to sequence every B cell in humans, so representative samples (generally derived from peripheral blood) are taken. The first human studies sought to discover frequencies of VDJ segment usage, the similarity in VDJ segment usage, and the CDR3 sequence between individuals, and to estimate the size of the antibody repertoire $[2,3,5,11,64-67]$. Size-estimates are difficult as samples taken are a small representation of the entire repertoire, but sequencing independent replicate libraries in a capture-recapture analysis indicates a minimum bound of $10^{6}$ unique $\mathrm{V}_{\mathrm{H}}$ rearrangements [2]. This estimate neglects size 
differences in the repertoire of different B cell subsets, so there are likely to be a greater number of unique naïve B cells, but fewer unique memory cells than this [4]. Comparing the repertoire across individuals showed that VDJ segments were used in unequal frequencies in the repertoire, but that their pattern of usage was similar between individuals, indicating inherent biases in the VDJ recombination process, and preferential use of core genes $[2,3,64]$. Despite similar VDJ usage though, there was limited overlap in the CDR3 repertoires between the two individuals studied by Arnaout et al. [3].

The antibody repertoire after vaccination has been examined using NGS for four different vaccines (Table 1). These studies have been able to broadly demonstrate minor changes in VDJ segment usage, and the size and diversity of the different B cell lineages after vaccination $[13,16]$. By comparing NGS data to sequences from known antigen-specific cells, it appears that the repertoire obtained by NGS includes antibody sequences that are targeted against vaccine antigens $[16,18]$. A recent study used $V_{H}$ sequence databases generated after vaccination to allow characterization of the serum antibody repertoire using highresolution liquid chromatography tandem mass spectrometry [68]. This technique has great potential for demonstrating the relationship between the $\mathrm{V}_{\mathrm{H}}$ sequence repertoire, and the repertoire of serum antibodies, but is currently limited by the requirement for individual sequence profiles on which to map the mass spectrometry data, so proteins represented in the serum, but not the sequence data, cannot be identified. Two studies assessed the difference between the trivalent-inactivated (TIV), and the live-attenuated influenza vaccine (LAIV), on the post-vaccination repertoire, and showed that TIV causes increased production of abundant IgG lineages compared to LAIV [4,16]. To determine whether age is a confounding factor in repertoire sequencing, two studies investigated differences in antibody repertoire with age, which are discussed later $[13,16]$. There remain several important challenges to making the most effective use of NGS data for studying vaccine responses. These are primarily related to the methodology used for sequencing/analysis (Box 2) and also our ability to reliably identify the small number of antigen-specific sequences from large datasets of the total repertoire (Figure 1). 


\section{Identification of the antigen-specific repertoire, and the use of vaccination as a model system}

The ability to distinguish between the total antibody repertoire, and antigen-specific antibody repertoire is key for any attempt to reliably utilize NGS data for elucidation of B cell responses to vaccination (Figure 1). Whereas NGS methods are well suited to studying perturbations in the total sampled repertoire, to date the low-resolution methods have provided the most insight into the antigen-specific repertoire after vaccination (Table 1 and [56]). To assess the full diversity of the antigen-specific repertoire, it is necessary to have methods for identification of antigen-specific sequences from total NGS repertoire data.

One way to identify such sequences is to search for those that have a degree of identity to previously described sequences, a technique that Zhu et al. used for de-novo identification of new VRC01 antibodies (from a new donor) against HIV [69]. Cross-donor phylogenetic analysis using known VRC01 sequences, and sequences from the new donor, was used to identify novel VRC01 $\mathrm{V}_{\mathrm{H}}$ sequences based on evolutionary similarity. $\mathrm{V}_{\mathrm{L}}$ sequences were identified based on the presence of a five AA motif present in the previously described sequences, and when reconstituted with the $\mathrm{V}_{\mathrm{H}}$ genes were able to neutralize HIV-1 with varying potency.

An a priori method for the identification of antigen-specific sequences from total repertoire data is by analysis of the antibody sequences that are shared between multiple individuals following recent exposure (through infection, immunization or autoimmunity) to a common antigen - this is termed the 'convergent repertoire'. A study of the antibody repertoire in the cerebrospinal fluid, and peripheral blood of six multiple sclerosis (MS) patients, revealed some minor biases in $\mathrm{V}_{\mathrm{H}}$ gene usage in the cerebrospinal fluid repertoire of the MS patients, which were not seen in the control patients [70]. However, it should be noted that only two $\underline{\mathrm{V}}_{\mathrm{H}_{-}}$genes were found at significantly greater levels in the MS patients, and the level of significance was low $(\mathrm{p}=0.04 / 0.01)$. A study comparing the antibody repertoire in 60 dengue patients during acute disease, and at convalescence, indicated the presence of convergent CDR3 signatures (predominantly 'ARLDYYYYYGMDL') between individuals which were enriched during acute disease compared to at convalescence or in healthy controls [47]. However, no work was subsequently done to confirm the 
specificity of these sequences for dengue antigens. The extent to which an individual's B cell repertoire for an antigen is shared with other individuals remains uncertain and is a key question to answer in order to determine the utility of 'convergent repertoire' analysis. Given the degree of antibody diversity highthroughput NGS methods will be invaluable in clarifying this important area.

In the analysis the of convergent repertoire, sequence similarity may not only be in the form of exact sequence matches, but also specific shared motifs within the sequences, such as CDR3 length, $\mathrm{V}_{\mathrm{H}}$ usage, and amino acid usage in key locations, which could require sophisticated algorithms for identification $[47,62]$. Vaccine studies are an ideal tool for investigating the degree to which convergent sequences are likely to be antigen-specific. The ability to control the timing of antigen administration and sampling in vaccine studies increases our ability to sample the repertoire at timepoints when there is maximum egress of antigen-specific cells into the sampled compartment. Generally, an antigen-specific plasma cell burst is seen on days 4-10 in the peripheral blood following vaccination (with different kinetics for a primary vs secondary response [7173]). If sampling is performed both before, and 4-10 days post-vaccination, filtering for sequences which increase, and persist at multiple timepoints after vaccination, in addition to analysis of convergence, would facilitate identification of antigen-specific sequences. The increase in sequence abundance will be even more pronounced if mRNA is used as the template (see Box 2, point 1), due to the high level of transcriptional activity in plasma cells [74]. In addition, FACS can be used to enrich for plasma cells or antigen-specific cells, so that a greater percentage of the sampled repertoire is antigen-specific (Figure 1). An additional consideration is that different B cell subsets have different kinetics after vaccination [23]. The majority of NGS studies have focused on sampling around day 7, where plasma cells are most likely to peak, yet with sufficient depth of sequencing it should also be possible to detect convergent repertoires related to antigenspecific memory cell populations present at later time points as well [23].

Vaccine studies which include both sequencing of the total repertoire, and the repertoire of sorted antigen-specific cells (or antigen-enriched plasma cells), will facilitate analysis of the extent to which antigen-specific sequences are shared (convergent or 'public' repertoire), compared to those antigen-specific sequences, which may be specific for a particular individual ('private' repertoire). Furthermore, functional 
characterization of the putatively antigen-specific sequences will demonstrate whether the most functional antibodies are predominantly present in the private, or shared part of the antigen-specific repertoire.

\section{Sequencing the antibody repertoire as a tool for vaccinology}

Antibody repertoire sequencing technology has potential applications in multiple areas of vaccine development, including measurement of vaccine response, predicting vaccine safety, and guiding the development of more effective vaccines by increasing our understanding of B cell and GC immunology.

\section{Generating mAbs}

Perhaps the most well defined use of antibody sequencing after vaccination is in the generation of mAbs. mAbs can be used for passive immunization against certain diseases [75], and have important research applications, including verification of methods for identification of antigen-specific sequences from NGS repertoire data. After antigen administration, plasma cells (or antigen-enriched cells) can be isolated from peripheral blood by FACS and sequenced to identify the sequences of the antibodies that are produced in response to the antigen. Generally, the most abundant sequences are then cloned into an expression vector for functional characterization. The requirement of both $V_{H}$ and $V_{L}$ sequences to be obtained for mAb generation resulted in initial studies using a single-cell sorting approach followed by $\mathrm{V}_{\mathrm{H}}$ and $\mathrm{V}_{\mathrm{L}}$ sequencing of individual cells [20,59]. As methods are developed for linking the $\mathrm{V}_{\mathrm{H}}: \mathrm{V}_{\mathrm{L}}$ repertoires (Box 2), it will be possible to identify mAbs in a more high-throughput manner (as demonstrated by Reddy et al. [76]). Despite the exact method used, such studies have demonstrated $6-76 \%[22,59,76,77]$ of cell lines created produce antigen-specific antibodies, resulting in a significant decrease in the time taken to generate effective mAbs compared to the conventional methods of screening large combinatorial libraries.

\section{Tracking known sequences}

There are numerous antibody sequences in the literature that have a known specificity (Table 1 and [56]). Incorporating repertoire sequencing into studies of vaccines, autoimmunity and infectious diseases will aid in the creation of increasingly comprehensive databases containing antibody sequences with defined specificity 
and function (Figure 2). In theory, if these databases contained sequences known to be specific for a certain vaccine antigen, we could search for these previously described sequences in the post-immunisation repertoire during new studies of the vaccine antigen, and infer the degree to which a protective immune response has been generated. Current data from a limited range of antigens suggest that a vaccine response is likely to be complex, and in-depth studies of the antibody repertoire will be required to fully understand the relationship between NGS data and immunogenicity, as discussed later. Although there are currently few known sequences for auto-reactive antibodies, the identification of known auto-reactive sequences in the post-vaccination repertoire, would be of interest in terms of signaling the potential for certain vaccines to increase the risk of autoimmune disease. However, the large number of 'self' epitopes, and the potential diversity of auto-reactive sequences that could target these epitopes make it unlikely that all potential autoreactive sequences could ever be identified. A recent study of the antibody repertoire in MS patients identified expansion of some sequences potentially related to the disease [70] (although this was not confirmed with functional assays), and as such sequences are discovered, it would be beneficial to routinely test for them in vaccine trials as a precautionary measure.

Considering a specific example of the utility of tracking known sequences for vaccine development, in the case of HIV, neutralizing antibodies have been discovered against the envelope glycoprotein (Env) in HIV-infected individuals, but stimulating formation of these antibodies by vaccination is difficult, as their germline precursors have poor affinity for Env [78,79]. In some HIV infected individuals, it appears that neutralizing antibodies may be formed from mutated, cross-reactive precursors that were initially generated during previous non-HIV infections, and then further mutate to become specific for Env [80]. It has been observed in one study, that the unmutated precursor of an effective anti-HIV antibody was able to bind to the founder HIV virus. Sequencing the viral Env and antibody genes at multiple timepoints after virus transmission indicated that antibody maturation was preceded by viral evolution, suggesting that such coevolution is a potential method for driving formation of anti-Env neutralizing antibodies [81]. It seems for HIV, that an effective vaccine requires prior identification of the correct germline precursor antibody sequence. The germline precursor could then be stimulated using an alternate immunogen to Env, and 
successive immunogens then administered to guide B cell maturation to form the desired final antibody [82]. The success of this work is being driven by the ability to identify low-abundance antibody sequences in high-depth antibody sequencing experiments [79].

\section{Investigating breadth of the antibody response}

As well as identifying specific sequences, NGS data can give insight into the breadth of the antibody repertoire (a measure of the diversity, in terms of the number and abundance of different sequence clones [83]) used in an immune response. For some vaccines, the antigenic site targeted is highly variable (HIV-1 Env/Plasmodium vivax Duffy-binding protein), or undergoes seasonal change (influenza HA); a broader response can potentially confer protection against more antigenic variants. Antibody repertoire sequencing has been instrumental in demonstrating that use of a Toll-like receptor (TLR) agonist in an oil-in-water adjuvanted malaria vaccine formulation is able to broaden the antibody response in mice. Use of the TLR agonist in the vaccine led to improved antigen neutralization, and efficacy against more varied malarial strains [84]. Similar observations have been made using oil-in-water adjuvanted influenza vaccines, which induced broadly cross-reactive responses against the HA head, providing some protection against drifted strains of the virus [85].

While it seems reasonable to suggest that the breadth of the antibody repertoire is a measure of ability to neutralize diverse antigenic strains, it is also possible that single antibodies can have broadly neutralizing activity by targeting conserved regions on otherwise variable antigens. This appears to be the case with influenza HA, where broad neutralization of multiple variants can arise from a clonal antibody response targeting the conserved stem region or a diverse response targeting the variable head region [86]. Stem-binding antibodies are not found after seasonal influenza vaccination, but of the 28 HA-binding mAbs generated from eight subjects after 2009 pandemic H1N1 influenza vaccination, Li et al. found that three of these were able to bind the stem region and elicit cross-reactive protection [86]. The authors hypothesize that as the influenza response is predominately driven by memory cells, and the pandemic virus has a highly divergent HA head compared to seasonal variants, only the cross-reactive memory cells would be stimulated. This is a contested issue though, as a study by Wrammert et al. in which over 50 influenza-specific mAbs 
were generated from plasma cell sequences after influenza vaccination showed that they exhibited the highest affinity for the current circulating strain of influenza [20]. The conflicting nature of these studies indicate that the high-throughput nature of NGS data would be better able to give insight into the true breadth of the response after seasonal influenza vaccination. Vollmers et al. have developed a novel method of barcoding individual RNA molecules, and building consensus reads to generate accurate antibody sequence data, which they used to demonstrate the appearance of some identical sequences after two different annual influenza vaccines in the same individuals (Table 1) [4]. Using conventional immunological assays in parallel with antibody repertoire sequencing would also be useful to improve our understanding of how repertoire data relates to functional measures of cross-reactivity.

\section{Understanding Immunological Mechanisms of Vaccination}

The majority of vaccines and immunization schedules were initially developed empirically, rather than through a detailed understanding of the immunological mechanisms of vaccination. Data generated from antibody repertoire sequencing both after vaccination, and in populations without vaccine challenge, is starting to give great insight into the underlying immunology of vaccination. For vaccines such as hepatitis B, where different dose schedules are available, the schedule used affects vaccine immunogenicity [87]. Using repertoire sequencing to improve understanding of the GC reaction and antibody maturation pathways could aid the design of optimal dose schedules. Booster doses of a vaccine are used to increase the number and affinity of antigen-specific antibodies. Using surface plasmon resonance to analyze the antibody repertoire after repeated doses of tetanus toxoid (TT) vaccination in two individuals, the limits of antibody affinity maturation were determined to be reached after just two doses for this antigen [22]. Sequencing plasma cells produced after these vaccinations indicated that the limit of somatic hypermutation was also reached after two doses. A third dose of TT did not increase either the affinity of the anti-TT antibodies, or the diversity of the repertoire [22]. Repeat doses of vaccine given close together may increase the magnitude of the antibody response by re-stimulating GC's, or by stimulating new GC's to form, resulting in the production of new plasma cells. Sorting and sequencing antigen-specific B cells present in the peripheral blood after vaccination may allow capture of recent GC emigrants. Observing the relative numbers of these 
cells, and their mutational load, could give insight into proliferation and affinity maturation within the GC after repeat vaccination. It would be instructive to understand the interaction between dose interval and antibody maturation; the repertoire may become more clonal after repeat doses due to selection in the GC. Dose schedules could then be altered accordingly to optimize responses, and prevent unnecessary vaccinations.

It is also important to consider that the total B cell repertoire is actually composed of a combination of repertoires from different B cell subsets [12,88-90], and that these different B cell subsets could be responding differently to vaccination [91]. To determine whether IgM memory cells were early emigrants from the T-dependent GC response (and so related to IgG memory cells), or were instead formed after stimulation with T-independent antigens, the repertoire in $\operatorname{IgM}$ memory and $\operatorname{IgG}$ memory cells was compared in a population with no vaccine challenge. Wu et al. and Briney et al. both observed differences in VDJ segment composition in the IgM and $\operatorname{IgG}$ memory repertoires $[88,89]$. Wu et al. also found the $\operatorname{IgM}$ repertoire to contain fewer negatively charged amino acids, and greater levels of tyrosine, as well as lower hydrophobicity and aliphatic index, supporting the notion that IgG and IgM memory cells could comprise populations with distinct origins, which have been formed through responses to different antigenic stimuli (although it cannot be ruled out that $\operatorname{IgG}$ and $\operatorname{IgM}$ memory cells have the same origins, but are subject to distinct regulatory mechanisms) [88]. However, by defining IgM memory by having a V gene mutation frequency of greater than $1 \%$, rather than by sorting CD27+ cells, Wang et al. found little difference between VDJ segment composition in $\operatorname{IgM}, \operatorname{IgD}, \operatorname{IgG}$ or IgA sequences [12]. This topic could clearly benefit from further study, and a clarification on the optimal definitions of how to sort cell subsets either physically before sequencing, or computationally from the sequence data. Nevertheless, it is clearly the case that cell subsets and isotype should be taken into account in studies of the antibody repertoire after vaccination.

\section{Measuring vaccine immunogenicity}

Developing new vaccines, and predicting efficacy of available vaccines in new populations requires measuring correlates of immunity. Correlates are generally measures of the immune response, such as antibody concentration, or function (e.g. serum bactericidal assay for group C N. meningitides or HA 
inhibition assay for influenza), which relate to vaccine-induced protection against infection. For many vaccines, such correlates are often unavailable, or hard to measure and standardize between laboratories [92]. Sequencing the antibody repertoire after vaccination can provide a detailed dissection of the vaccine-induced B cells underlying the antibody response, and a potential application of this is in deriving novel correlates of protection. Although repertoire data can be used to identify known antigen-specific sequences, and the breadth of the response, it remains to be demonstrated whether it can be used as a valid standalone measure of immunogenicity. A critical first step is to distinguish the vaccine-specific repertoire from the total repertoire, and as discussed, this is increasingly feasible. The vaccine-specific repertoire (in terms of number and abundance of sequences) would then need to be correlated with a known indicator of vaccine response, or with protection from infection. Current NGS depth and cost (Box 1) makes it an increasingly feasible proposition to carry out repertoire sequencing in conjunction with routine clinical tests on a scale compatible with that of clinical vaccine trials necessary for investigating this application. Recent methods allowing highthroughput identification of serum antibodies will also help to show to which antigen-specific sequences ultimately give rise to long-lived plasma cells and mediate long-term protection $[52,68]$. This technique has so far been used to investigate the serum antibody repertoire in two individuals after TT vaccination, and showed that $<5 \%$ of antigen-specific plasma cell sequences identified 7 days post-vaccination, are found to encode antibodies that are also present in the serum response 9 months post-vaccination at sufficient levels to be detected [68]. Other aspects that must be considered for a good correlate of immunity are that the signal detected is not transient, and that it is not confounded by other individual factors such as genotype, antigen exposure history, and age. Signal persistence in antibody repertoire studies has not been well investigated. It is clear that perturbations can be seen on days 7,8 and 14 after vaccination (Table 1), and some sequences are found to persist in individuals for at least a year, and are identifiably re-stimulated upon further vaccination [4]. It is less clear to what degree such perturbations in the total repertoire reflect perturbations in the antigen-specific repertoire, but as methods for identifying the antigen-specific repertoire improve (Figure 1), this should become clearer. 
The potential confounding effects of genotype, antigen exposure history, and age have been investigated to different degrees. By studying the antibody repertoire in two pairs of monozygotic twins, Glanville at al. investigated the relative influence of genetic and environmental factors in structuring the naïve repertoire [11]. Although limited to just four individuals, they found that certain $V_{H}$ and $D_{H}$ segments were used at significantly different frequencies between the different twins compared to within twin pairs, indicating that individual genetic differences should be taken into account when studying changes in VDJ segment usage after vaccination. There was limited overlap in the CDR3 repertoires both between and within twins, which may be due to limitations in sequencing depth (so there may be more overlap if more sequences were obtained), but is indicative of the random nature of VDJ junctional diversification during B cell development and somatic hypermutation during antigen-response, which drive diversification of the antibody repertoire. An additional complication is that the genotype at other loci as well as the immunoglobulin locus may also affect the antibody repertoire, as has been shown by the impact of an individuals human leukocyte antigen locus genotype on their $\mathrm{T}$ cell receptor repertoire [93]. It would be of interest to conduct a similar study to investigate the relationship between features of the antibody repertoire (such as VDJ composition), and genotype at other immunologically important loci.

As well as varying between individuals, vaccine response varies with age [94], but the reasons for this are not fully known. Jiang et al. studied the antibody repertoire before and after influenza vaccination in children (8-17 years), young adults (18-30 years) and the elderly (70-100 years) [14]. As there were only four individuals in the elderly age group, statistical comparisons were not performed, but this study did show that in two of the elderly individuals, the repertoire was highly clonal and had a greater mutational load compared to the younger individuals. In another study, the isotype-specific antibody repertoire was studied after simultaneous administration with pneumococcal and influenza vaccination in six younger (19-45 years) and six older (70-89 years) individuals [13]. There was a slight increase in IgG mutation in the older age group, but the most striking differences were in the $\operatorname{IgA}$ and $\operatorname{IgM}$ repertoires, which displayed slower clonal expansion as well as less mutation, and longer CDR3 regions in the elderly. Even with no vaccine stimulation, there appear to be age-related differences in the naïve repertoire, with elderly individuals having 
different VDJ recombination frequencies [95], longer CDR3 regions, greater persistence of large clones, and increased mutational loads [12] compared to younger individuals. Interestingly, sex does not appear to effect the repertoire, despite the presence of other known sex-specific effects on the immune system [96]. Hence, it is clear that there are age-related and isotype-dependent effects on the repertoire, but it is necessary to conduct larger vaccine trials in individuals of different ages (including infants, which have been previously omitted), so that statistical comparisons can be made to confirm these findings.

Considering antigen exposure history, the potential effect of previous influenza vaccination on subsequent influenza vaccinations has been discussed, indicating the importance of documenting vaccine and disease history where possible. In addition to previous infections impacting how the repertoire responds to vaccination, the effect of chronic infection needs to be considered. Wang et al. documented chronic CMV and EBV infection status in a repertoire study of 27 individuals over two years [12]. CMV infection resulted in increased $\mathrm{V}_{\mathrm{H}}$ mutation in the $\mathrm{IgG}$ and $\mathrm{IgM}$ repertoire, while $\mathrm{EBV}$ infection resulted in an increased number of persistent clonal groups in the repertoire Neither infection resulted in altered VDJ gene segment usage in the repertoire, but segment usage does appear to be different between healthy donors, patients with chronic hepatitis $\mathrm{C}$ infection, and in patients who have recovered from hepatitis $\mathrm{C}$ infection [45]. As the number of antigens an individual has been exposed to, and the rate of chronic viral infection increase with age, the effect of antigen exposure history, and age on the antibody repertoire will be tightly linked.

So, although antibody repertoire sequencing has promise as a novel tool to measure vaccine immunogenicity, more work is first required in order to improve and validate methods for identifying the antigen-specific repertoire (Figure 1). It is also essential that repertoire sequencing studies are conducted on larger sample sets so that more robust statistical comparisons can be made to investigate the effect of age, antigen exposure history and genotype, as well as to allow correlations to be drawn between repertoire data, and known correlates of immunity. 


\section{Concluding remarks}

Understanding of the antibody repertoire has increased enormously over the last decade, due mainly to improved sequencing technology. There is an increasingly comprehensive view of the structure of the baseline antibody repertoire, and how this differs between individuals and B cell subsets. An appreciation of how vaccine antigens dynamically restructure the antibody repertoire, and how certain adjuvants affect this process is also emerging.

Laboratory and bioinformatic techniques available for studying the antibody repertoire are constantly improving. Major advances in the last year now allow high-throughput identification of paired $\mathrm{V}_{\mathrm{H}}: \mathrm{V}_{\mathrm{L}}$ sequences [18], quantification of PCR-related bias and mutation [4], and identification of putatively antigen-specific sequences through repertoire convergence [47]. As groups working on vaccine development increasingly use repertoire sequencing, it is important that these different methods are synthesized, and a 'gold standard' sequencing protocol agreed so that data generated from different laboratories can be reliably compared. To make full use of available data, the creation of an open-access database for the accumulation of repertoire data after vaccination, natural infection and in autoimmune disease needs to be considered (Figure 2). Researchers can then concentrate on how to reliably interpret and make practical use of the resulting data, in the face of the potential confounding factors of genotype, age and antigen exposure history.

Analysis of the post-vaccination antibody repertoire is providing a fascinating view of the B cell response to vaccines. There is a compelling case that the practical application of these data will have a profound affect on the development of new vaccines. The use of vaccines as a model system for studying the repertoire also prefigures the use of repertoire sequencing technology in the more complex immune responses apparent during autoimmunity and disease.

\section{Acknowledgements}

J.D.G. receives funding from the BBSRC and GlaxoSmithKline in the form of an iCASE studentship. J.T. is a James Martin Fellow and was supported by an ESPID Fellowship Award. A.J.P. is a Jenner Investigator 
and James Martin Senior Fellow. A.J.P. \& D.F.K are supported by the NIHR Oxford Biomedical Research Centre.

\section{Competing interests}

A.J.P. acts as an investigator on behalf of Oxford University for clinical trials of vaccines for which the University receives grants from Pfizer, Novartis, GlaxoSmithKline and Sanofi Pasteur MSD, but does not receive any personal payments from them. J.T. and D.F.K. have received support from pharmaceutical companies to attend scientific meetings.

\section{References}

1 Ehreth, J. (2003) The global value of vaccination. Vaccine 21, 596-600

2 Boyd, S.D. et al. (2009) Measurement and Clinical Monitoring of Human Lymphocyte Clonality by Massively Parallel V-D-J Pyrosequencing. Sci. Transl. Med. 1, 12ra23-12ra23

3 Arnaout, R. et al. (2011) High-resolution description of antibody heavy-chain repertoires in humans. PLoS One $6, \mathrm{e} 22365$

4 Vollmers, C. et al. (2013) Genetic measurement of memory B-cell recall using antibody repertoire sequencing. Proc.Natl.Acad.Sci. U.S.A. 110, 13463-8

5 Glanville, J. et al. (2009) Precise determination of the diversity of a combinatorial antibody library gives insight into the human immunoglobulin repertoire. Proc. Natl. Acad.Sci. U.S.A. 106, 20216-21

6 Roux, K.H. (1999) Immunoglobulin structure and function as revealed by electron microscopy. Int. Arch . Allergy Immunol. 120, 85-99

7 North, B. et al. (2011) A new clustering of antibody CDR loop conformations. J. Mol. Biol. 406, 228-256

8 Tonegawa, S. (1983) Somatic Generation of Antibody Diversity. Nature 302, 575-581

9 Victora, G.D. and Nussenzweig, M.C. (2012) Germinal centers. Annu. Rev. Immunol. 30, 429-57

10 Shih, H. and Krangel, M.S. (2013) Chromatin Architecture, CCCTC-Binding Factor, and V(D)J Recombination: Managing Long-Distance Relationships at Antigen Receptor Loci. J. Immunol. 190, 49154921

11 Glanville, J. et al. (2011) Naive antibody gene-segment frequencies are heritable and unaltered by chronic lymphocyte ablation. Proc. Natl. Acad. Sci. U.S.A. 108, 20066-71

12 Wang, C. et al. (2013) Effects of Aging, Cytomegalovirus Infection, and EBV Infection on Human B Cell Repertoires. J. Immunol. DOI: 10.4049/jimmunol.1301384 
14 Ademokun, A. et al. (2011) Vaccination-induced changes in human B-cell repertoire and pneumococcal IgM and IgA antibody at different ages. Aging Cell 10,922-30

Jiang, N. et al. (2013) Lineage structure of the human antibody repertoire in response to influenza vaccination. Sci.Transl. Med. 5, 171ra19

Boyd, S.D. et al. (2013) Human lymphocyte repertoires in ageing. Curr. Opin. Immunol. 25, 511-5

Dekosky, B.J. et al. (2013) High-throughput sequencing of the paired human immunoglobulin heavy and light chain repertoire. Nat. Biotechnol. 31, 166-169

Krause, J.C. et al. (2011) Epitope-specific human influenza antibody repertoires diversify by B cell intraclonal sequence divergence and interclonal convergence. J. Immunol. 187, 3704-11

Wrammert, J. et al. (2008) Rapid cloning of high-affinity human monoclonal antibodies against influenza virus. Nature 453, 667-71

Tan, Y.-C. et al. (2014) High-throughput sequencing of natively paired antibody chains provides evidence for original antigenic sin shaping the antibody response to influenza vaccination. Clin. Immunol. 151, 55-65

Poulsen, T.R. et al. (2011) Limits for antibody affinity maturation and repertoire diversification in hypervaccinated humans. J. Immunol. 187, 4229-35

Frölich, D. et al. (2010) Secondary immunization generates clonally related antigen-specific plasma cells and memory B cells. J. Immunol. 185, 3103-3110

Adderson, E. et al. (1993) Restricted immunoglobulin VH usage and VDJ combinations in the human response to Haemophilus influenzae type b capsular polysaccharide. J. Clin. Invest. 91, 2734-2743

Liu, L. and Lucas, A.H. (2003) IGH V3-23*01 and its allele V3-23*03 differ in their capacity to form the canonical human antibody combining site specific for the capsular polysaccharide of Haemophilus influenzae type b. Immunogenetics 55, 336-8

Rohatgi, S. et al. (2009) Molecular dissection of antibody responses against pneumococcal surface protein A: evidence for diverse DH-less heavy chain gene usage and avidity maturation. J. Immunol. 182, 5570-85

Wrammert, J. et al. (2011) Broadly cross-reactive antibodies dominate the human B cell response against 2009 pandemic H1N1 influenza virus infection. J. Exp. Med. 208, 181-93

$\mathrm{Yu}$, X. et al. (2008) Neutralizing antibodies derived from the B cells of 1918 influenza pandemic survivors. Nature 455, 532-6

Krause, J.C. et al. (2012) Human monoclonal antibodies to pandemic 1957 H2N2 and pandemic 1968 H3N2 influenza viruses. J. Virol. 86, 6334-40 

of influenza virus hemagglutinin. Proc. Natl. Acad. Sci. U. S. A. 108, 14216-21

Corti, D. et al. (2011) A neutralizing antibody selected from plasma cells that binds to group 1 and group 2 influenza A hemagglutinins. Science 333, 850-6

Weitkamp, J.-H. et al. (2003) Infant and adult human B cell responses to rotavirus share common immunodominant variable gene repertoires. J. Immunol. 171, 4680-8

Weitkamp, J.-H. et al. (2003) Generation of recombinant human monoclonal antibodies to rotavirus from single antigen-specific B cells selected with fluorescent virus-like particles. J. Immunol. Methods 275, 223-237

Weitkamp, J.-H. et al. (2005) Natural evolution of a human virus-specific antibody gene repertoire by somatic hypermutation requires both hotspot-directed and randomly-directed processes. Hum. Immunol. 66, 666-76

Tian, C. et al. (2008) Immunodominance of the VH1-46 antibody gene segment in the primary repertoire of human rotavirus-specific B cells is reduced in the memory compartment through somatic mutation of nondominant clones. J. Immunol. 180, 3279-88

Wu, X. et al. (2011) Focused evolution of HIV-1 neutralizing antibodies revealed by structures and deep sequencing. Science (80-. ). 333, 1593-602

Zhu, J. et al. (2012) Somatic Populations of PGT135-137 HIV-1-Neutralizing Antibodies Identified by 454 Pyrosequencing and Bioinformatics. Front. Microbiol. 3, 315

Scheid, J.F. et al. (2009) Broad diversity of neutralizing antibodies isolated from memory B cells in HIVinfected individuals. Nature 458, 636-40

Zhu, J. et al. (2013) Mining the antibodyome for HIV-1 - neutralizing antibodies with next-generation sequencing and phylogenetic pairing of heavy / light chains. DOI: 10.1073/pnas.1219320110

Huang, C. et al. (2004) Structural basis of tyrosine sulfation and VH-gene usage in antibodies that recognize the HIV type 1 coreceptor-binding site on gp120. Proc. Natl. Acad. Sci. U. S. A. 101, 2706-11

Bonsignori, M. et al. (2011) Analysis of a clonal lineage of HIV-1 envelope V2/V3 conformational epitopespecific broadly neutralizing antibodies and their inferred unmutated common ancestors. J. Virol. 85, 999810009

Corti, D. et al. (2010) Analysis of memory B cell responses and isolation of novel monoclonal antibodies with neutralizing breadth from HIV-1-infected individuals. PLoS One 5, e8805

Scheid, J.F. et al. (2011) Sequence and structural convergence of broad and potent HIV antibodies that mimic CD4 binding. Science 333, 1633-7

Racanelli, V.et al. (2011) Antibody V(h) repertoire differences between resolving and chronically evolving hepatitis $\mathrm{C}$ virus infections. PLoS One 6, e25606

Lu, D.R. et al. (2014) Identification of functional anti-Staphylococcus aureus antibodies by sequencing patient plasmablast antibody repertoires. Clin. Immunol. DOI: 10.1016/j.clim.2014.02.010 

reactive antibodies endowed with neutralizing and enhancing activity. Cell Host Microbe 8, 271-83

Smith, S. a et al. (2012) Persistence of circulating memory B cell clones with potential for dengue virus disease enhancement for decades following infection. J. Virol. 86, 2665-75

De Alwis, R. et al. (2011) In-depth analysis of the antibody response of individuals exposed to primary dengue virus infection. PLoS Negl. Trop. Dis. 5, e 1188

Dejnirattisai, W. et al. (2010) Cross-reacting antibodies enhance dengue virus infection in humans. Science 328 , 745-8

Wine, Y.et al. (2013) Molecular deconvolution of the monoclonal antibodies that comprise the polyclonal serum response. Proc. Natl. Acad.Sci. U.S.A. 110, 2993-8

Awdeh, Z.L. et al. (1968) Isoelectric Focusing in Polyacrylamide Gel and its Application to Immunoglobulins. Nature 219, 66-67

Seidman, J.G. et al. (1978) Multiple related immunoglobulin variable-region genes identified by cloning and sequence analysis. Proc. Natl. Acad. Sci. U.S.A. 75, 3881-5

Adderson, E.E. et al. (1991) Restricted Ig H chain V gene usage in the human antibody response to

Haemophilus influenzae type b capsular polysaccharide. J. Immunol. 147, 1667-74

Wilson, P.C. and Andrews, S.F. (2012) Tools to therapeutically harness the human antibody response. Nat. Rev. Immunol. 12, 709-719

Wang, X. and Stollar, B.D. (2000) Human immunoglobulin variable region gene analysis by single cell RTPCR. J. Immunol. Methods 244, 217-25

Tiller, T. et al. (2008) Efficient generation of monoclonal antibodies from single human B cells by single cell RT-PCR and expression vector cloning. J. Immunol. Methods. 329, 112-124

Smith, K. et al. (2009) Rapid generation of fully human monoclonal antibodies specific to a vaccinating antigen. Nat. Protoc. 4, 372-84

Lucas, A.H. et al. (1994) Variable region sequences of a protective human monoclonal antibody specific for the Haemophilus influenzae type b capsular polysaccharide. Infect. Immun. 62, 3873-80

Pinchuk, G. V et al. (1995) Predominant V-region gene configurations in the human antibody response to Haemophilus influenzae capsule polysaccharide. Scand. J. Immunol. 41, 324-30

Lucas, A.H. and Reason, D.C. (1999) Polysaccharide vaccines as probes of antibody repertoires in man. Immunol. Rev. 171, 89-104

Weinstein, J.A. et al. (2009) High-throughput sequencing of the zebrafish antibody repertoire. Science (80-. ). 324, 807-10 gene rearrangements. J. Immunol. 184, 6986-92 

repertoire is selected based upon its amino acid content. J. Immunol. 190, 5567-77

67 Briney, B.S. et al. (2012) Frequency and genetic characterization of V(DD)J recombinants in the human peripheral blood antibody repertoire. Immunology 137, 56-64

Lavinder, J.J. et al. (2014) Identification and characterization of the constituent human serum antibodies elicited by vaccination. Proc. Natl. Acad. Sci. DOI: 10.1073/pnas.1317793111

Zhu, J. et al. (2013) De novo identification of VRC01 class HIV-1-neutralizing antibodies by next-generation sequencing of B-cell transcripts. Proc. Natl. Acad.Sci. U.S.A. DOI: 10.1073/pnas.1306262110

Büdingen, H. Von and Kuo, T. (2012) B cell exchange across the blood-brain barrier in multiple sclerosis. J. Clin. Invest. 122, 4533-4543

Blanchard-Rohner, G. et al. (2009) Appearance of peripheral blood plasma cells and memory B cells in a primary and secondary immune response in humans. Blood 114, 4998-5002

Henn, A.D. et al. (2013) High-resolution temporal response patterns to influenza vaccine reveal a distinct human plasma cell gene signature. Sci.Rep. 3, 2327

Mitchell, R. et al. (2014) Polysaccharide-specific B cell responses to vaccination in humans. Hum. Vaccin. Immunother. 10, 0-1

Klein, U.et al. (1997) Evidence for a large compartment of IgM-expressing memory B cells in humans. Blood $89,1288-98$

Keller, M. a and Stiehm, E.R. (2000) Passive immunity in prevention and treatment of infectious diseases. Clin. Microbiol.Rev. 13, 602-14

Reddy, S.T. et al. (2010) Monoclonal antibodies isolated without screening by analyzing the variable-gene repertoire of plasma cells. Nat. Biotechnol. 28, 965-9

Smith, K. et al. (2013) Fully human monoclonal antibodies from antibody secreting cells after vaccination with Pneumovax ${ }^{\circledR} 23$ are serotype specific and facilitate opsonophagocytosis. Immunobiology 218, 745-54

Xiao, X. et al. (2009) Germline-like predecessors of broadly neutralizing antibodies lack measurable binding to HIV-1 envelope glycoproteins: implications for evasion of immune responses and design of vaccine immunogens. Biochem. Biophys. Res. Commun. 390, 404-9

Kwong, P.D. et al. (2013) Broadly neutralizing antibodies and the search for an HIV-1 vaccine: the end of the beginning. Nat. Rev. Immunol. 13, 693-701

Liao, H.-X. et al. (2011) Initial antibodies binding to HIV-1 gp41 in acutely infected subjects are polyreactive and highly mutated. J. Exp. Med.208, 2237-49

Liao, H.-X. et al. (2013) Co-evolution of a broadly neutralizing HIV-1 antibody and founder virus. Nature 496, 469-76 

Transl. Med. 3, 93ra69

Khurana, S. et al. (2011) MF59 adjuvant enhances diversity and affinity of antibody-mediated immune response to pandemic influenza vaccines. Sci. Transl. Med. 3, 85ra48

Li, G.-M. et al. (2012) Pandemic H1N1 influenza vaccine induces a recall response in humans that favors broadly cross-reactive memory B cells. Proc. Natl. Acad. Sci. U.S.A. 109, 9047-52

Jilg, W. et al. (1989) Vaccination against hepatitis B: comparison of three different vaccination schedules. $J$. Infect. Dis. 160, 766-9

Wu, Y.-C. et al. (2010) High-throughput immunoglobulin repertoire analysis distinguishes between human IgM memory and switched memory B-cell populations. Blood 116, 1070-8

Briney, B.S. et al. (2012) High-throughput antibody sequencing reveals genetic evidence of global regulation of the naïve and memory repertoires that extends across individuals. Genes Immun. 13, 469-73

Mroczek, E.S. et al. (2014) Differences in the Composition of the Human Antibody Repertoire by B Cell Subsets in the Blood. Front. Immunol. 5, 1-14

Tarlinton, D. and Good-Jacobson, K. (2013) Diversity among memory B cells: origin, consequences, and utility. Science 341, 1205-11

Plotkin, S.A. (2008) Vaccines: correlates of vaccine-induced immunity. Clin. Infect. Dis. 47, 401-9

Warren, R. et al. (2011) Exhaustive T-cell repertoire sequencing of human peripheral blood samples reveals signatures of antigen selection and a directly measured repertoire size of at least of at least 1 million clonotypes. Genome Res. 21, 790-797

Perrett, K.P. et al. (2010) Antibody persistence after serogroup C meningococcal conjugate immunization of United Kingdom primary-school children in 1999-2000 and response to a booster: a phase 4 clinical trial. Clin. Infect. Dis. 50, 1601-10

Rubelt, F. et al. (2012) Onset of immune senescence defined by unbiased pyrosequencing of human immunoglobulin mRNA repertoires. PLoS One 7, e49774

Markle, J.G. and Fish, E.N. (2013) SeXX matters in immunity. Trends Immunol. DOI: 10.1016/j.it.2013.10.006

Kolibab, K. et al. (2005) Immune Response to Pneumococcal Polysaccharides 4 and 14 in Elderly and Young Adults : Analysis of the Variable Heavy Chain Repertoire. Infect. Immun. 73, 7465-76

Laserson, U. et al. (2014) High-resolution antibody dynamics of vaccine-induced immune responses. Proc. Natl. Acad. Sci. U.S. A. DOI: 10.1073/pnas.1323862111

100 Gao, F. et al. (2013) Characterizing immunoglobulin repertoire from whole blood by a personal genome sequencer. PLoS One 8, e75294

101 Bolotin, D. a et al. (2012) Next generation sequencing for TCR repertoire profiling: Platform-specific features and correction algorithms. Eur. J. Immunol. DOI: 10.1002/eji.201242517 
102 Loman, N.J. et al. (2012) Performance comparison of benchtop high-throughput sequencing platforms. Nat. Biotechnol. 30, 434-9

103 Benichou, J. et al. (2012) Rep-Seq: uncovering the immunological repertoire through next-generation sequencing. Immunology 135, 183-91

104 Baum, P.D.et al. (2012) Wrestling with the repertoire: The promise and perils of next generation sequencing for antigen receptors. Eur. J. Immunol. 42, 2834-9

105 Matsuda, F. et al. (1998) The Complete Nucleotide Sequence of the Human Immunoglobulin Heavy Chain Variable Region Locus. J. Exp. Med. 188, 2151-2162

106 Zhu, J.et al. (2013) Mining the antibodyome for HIV-1-neutralizing antibodies with next-generation sequencing and phylogenetic pairing of heavy/light chains. Proc. Natl. Acad. Sci. U.S.A. 110, 6470-5

107 Kidd, B. a et al. (2014) Unifying immunology with informatics and multiscale biology. Nat. Immunol. 15, 118127 
Figure 1. Using vaccines to investigate the antigen-specific antibody repertoire. Vaccination will cause an increase in the number antigen-specific cells in the repertoire responding to the vaccine. There are then three ways in which antigen-specific sequences can be enriched for in the sampled repertoire, either physically or during analysis. 1) Identification of sequences that have a relative increase, and persist over time in the sampled repertoire after vaccination. 2) Physical enrichment using FACS to isolate plasma cells or antigen-specific cells for analysis, so that the sampled repertoire contains a greater proportion of antigen-specific sequences. 3) Identification of sequences shared by more than one individual (convergent repertoire), after stimulation with the same antigen. Using a combination of these three methods will likely give the greatest enrichment of antigenspecific sequences for subsequent analysis.

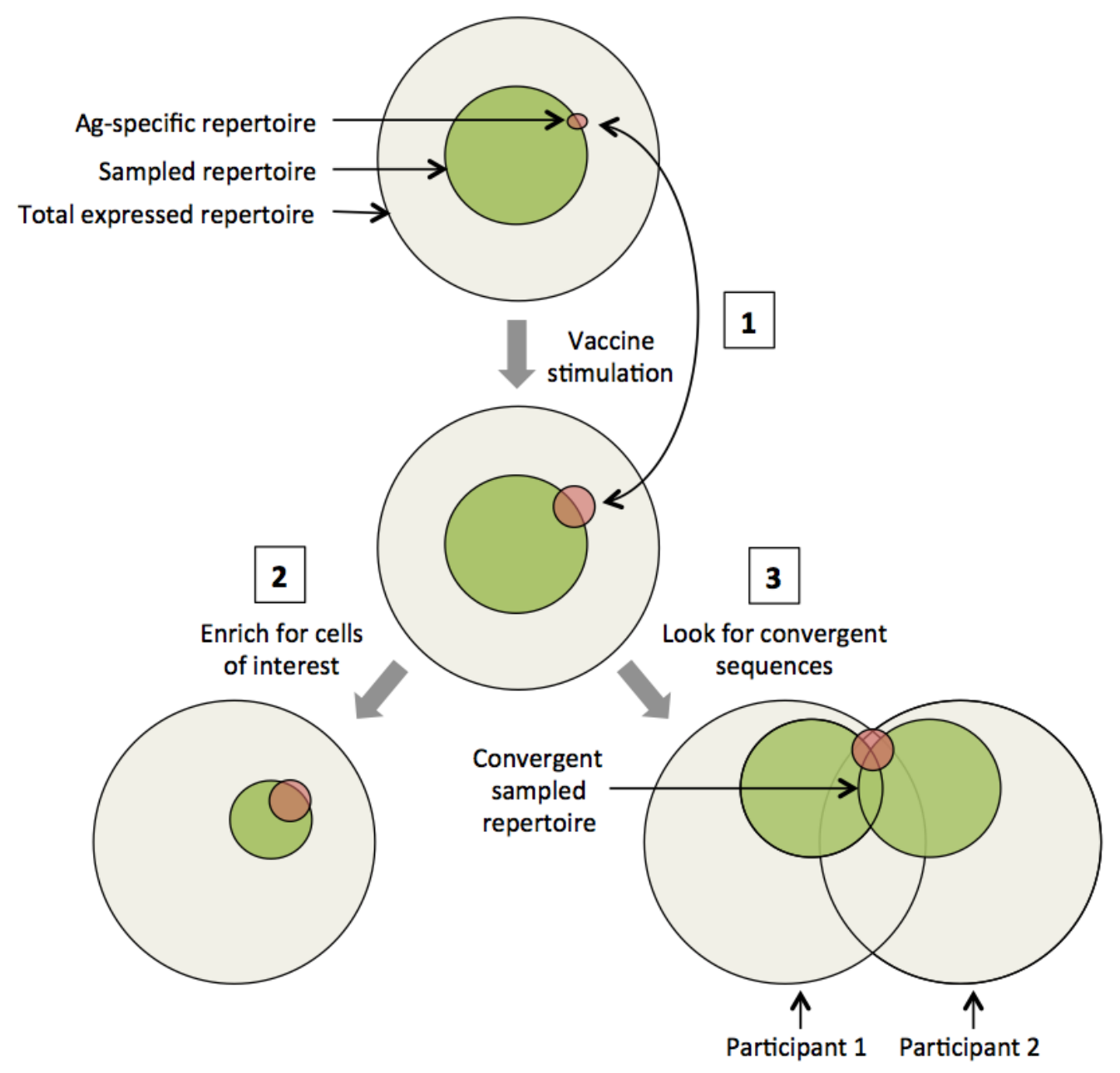


Figure 2. Proposed flow of information from repertoire sequencing projects to aid in the generation of data with practical uses. For such data to be suitable for meta-analysis, it is also important that a standardized sequencing protocol is used.

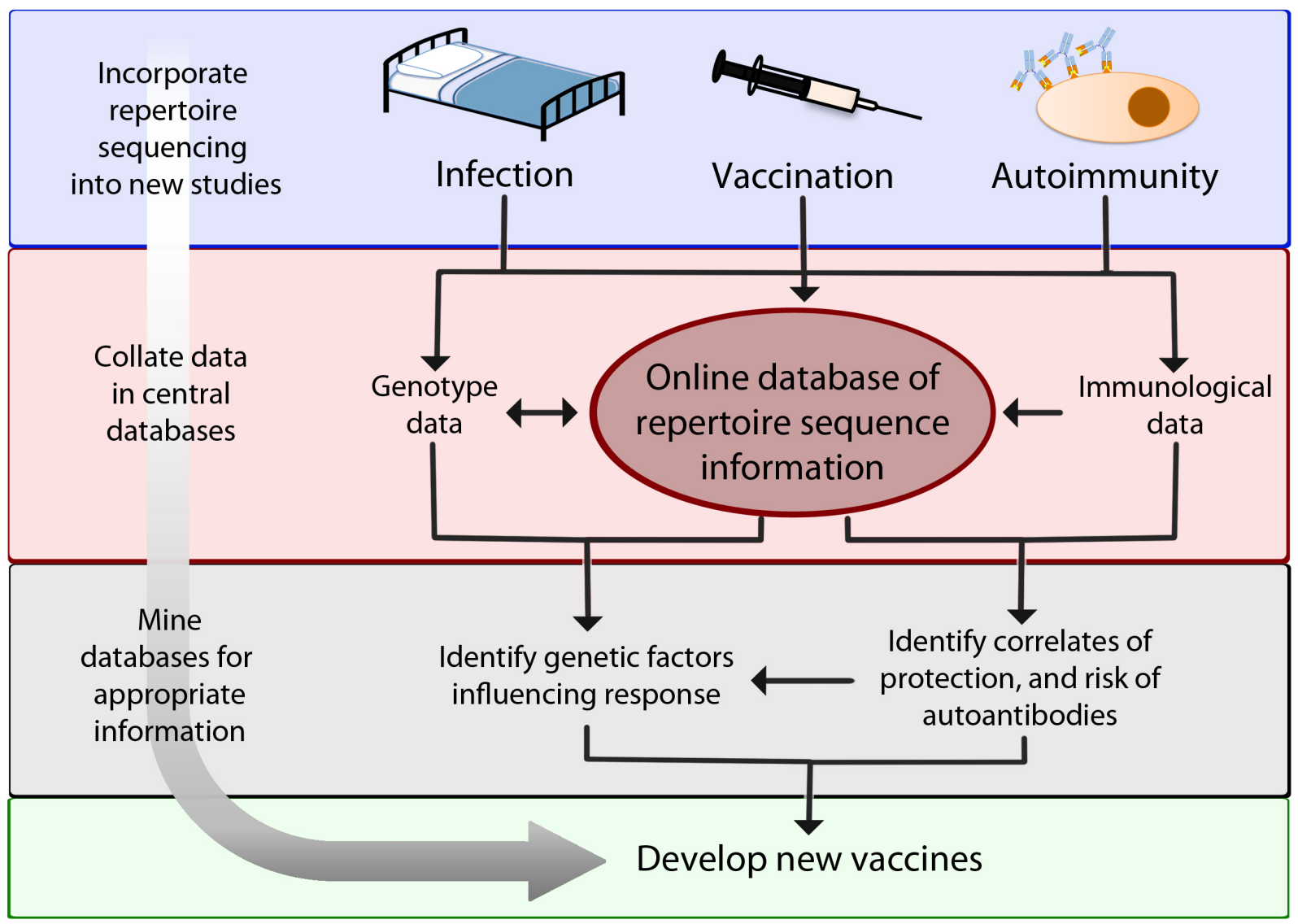


Box 1. Sequencing platforms used to study the antibody repertoire

The use of Sanger sequencing for characterization of single antibody genes from small numbers of cells is highly robust, as there is a high signal-to-noise ratio, low error rates, and a long read length. For characterizing whole repertoires of antibody genes from large numbers of cells, Sanger sequencing is too labor intensive, so NGS is used instead. NGS platforms allow multiple different DNA fragments to be sequenced simultaneously but generally have higher error rates, and shorter read lengths compared to Sanger sequencing. There are a number of different NGS platforms currently on the market, each utilizing different template amplification strategies, sequencing chemistries, and detection methods [99]. This leads to different types and degrees of sequence error (indels and substitutions) in the output data from each platform as well as different limitations such as read length, depth of sequencing (number of sequences that can be sequenced simultaneously), and cost.

High-throughput antibody repertoire sequencing was initially conducted using Roche's 454 sequencing technology, as this was the only platform with a sufficient read length ( $\sim 500 \mathrm{bp})$ to cover the entire $\mathrm{V}_{\mathrm{H}}$ region $[3,64]$. Recent advances in the Illumina chemistry now permit 300 bp paired-end sequencing (http://www.illumina.com/systems/sequencing.ilmn, accessed April 2014) on their MiSeq platform, so more laboratories are now moving towards this technology instead $[4,18]$. The large output from Illumina sequencing means that 96 samples can be multiplexed in a single run, while still giving more than 100,000 reads for each sample, thus offering significant cost benefits over 454 sequencing. Recently, the Ion Torrent has also been used to sequence the antibody repertoire, where the emphasis was on speed of data acquisition rather than depth of sequencing [100]. By using whole blood rather than sorted B cells to characterize the repertoire, the authors were able to generate sequence data from a sample in a single day using the Ion Torrent. Illumina, 454 and Ion Torrent, were recently compared by Bolotin et al. for their application in profiling the T cell receptor repertoire [101]. Data from this study was used to estimate the error rate of each of these platforms, as shown below. Costs are derived from [102].

\begin{tabular}{l|l|l|l|l|l} 
Platform & Mechanism & Error rate/type & Cost/Mb & Read Length & Depth \\
\hline
\end{tabular}




\begin{tabular}{|c|c|c|c|c|c|}
\hline 454 & Pyrosequencing & $\begin{array}{l}1.4 \% \text { of reads } \\
\text { homopolymer associated } \\
\text { indels }\end{array}$ & $\sim \$ 331$ & $500 \mathrm{bp}$ & $10^{5}-10^{6}$ reads \\
\hline Illumina & $\begin{array}{l}\text { Dye terminator } \\
\text { sequencing }\end{array}$ & $\begin{array}{l}3.2 \% \text { of reads } \\
\text { random substitutions }\end{array}$ & $\sim \$ 0.5$ & $2 \times 300 b p$ & $>10^{7}$ reads \\
\hline $\begin{array}{l}\text { Ion Torrent } \\
\text { (314 chip) }\end{array}$ & $\begin{array}{l}\text { Semiconductor } \\
\text { sequencing }\end{array}$ & $\begin{array}{l}1.2 \% \text { of reads } \\
\text { homopolymer associated } \\
\text { indels }\end{array}$ & $\sim \$ 22.5$ & $400 \mathrm{bp}$ & $10^{5}-10^{6}$ reads \\
\hline
\end{tabular}

NGS technology is rapidly advancing, with the major vendors frequently releasing updates to their systems, which reduce error and increase read length and depth. Costs are also constantly decreasing, making these technologies more accessible for use by laboratories with different specializations. As the technology develops, allowing deeper sequencing of the antibody repertoire, it is likely that more of the potential applications of this technology will be realized. 
Table 1. Summary of publications where the antibody repertoire has been investigated after vaccination.

\begin{tabular}{|c|c|c|c|c|}
\hline Vaccine & Cells used & Methodology & Key findings & Ref. \\
\hline \multicolumn{5}{|c|}{ Publications where the antibody repertoire has been investigated using a low-resolution approach } \\
\hline \multicolumn{5}{|l|}{ Influenza } \\
\hline TIV & $\begin{array}{l}\text { IgG plasmablasts } 7 \\
\text { days after } \\
\text { vaccination }\end{array}$ & $\begin{array}{l}\text { Single-cell } \mathrm{V}_{\mathrm{H}} \text { and } \mathrm{V}_{\mathrm{L}} \\
\text { PCR followed by } \\
\text { Sanger sequencing }\end{array}$ & $\begin{array}{l}\text { - Produced } 50 \text { mAbs from } 14 \text { individuals, against three different influenza } \\
\text { strains. } \\
\text { - Showed that the influenza-specific antibody response is pauci-clonal, with } \\
\text { extensive intraclonal diversification of the influenza-specific lineages due to } \\
\text { somatic hypermutation. }\end{array}$ & [20] \\
\hline TIV & $\begin{array}{l}\text { IgA- IgM- } \\
\text { plasmablasts } 7 \\
\text { days after } \\
\text { vaccination }\end{array}$ & $\begin{array}{l}\text { Single-cell } \mathrm{V}_{\mathrm{H}} \text { and } \mathrm{V}_{\mathrm{L}} \\
\text { PCR (including unique } \\
\text { barcodes for each cell), } \\
\text { followed by } 454 \\
\text { sequencing }\end{array}$ & $\begin{array}{l}\text { - Analyzed 384-768 sequences from each of three individuals. } \\
\text { - Cloned } 8 \text { mAbs from large clonal sequence families, and } 12 \text { mAbs from } \\
\text { singleton sequences. } 75 \% \text { of these mAbs bound and neutralized influenza, but } \\
\text { those from large clonal families were most effective. Three of these mAbs } \\
\text { bound more effectively to HA from previous influenza seasons than the HA } \\
\text { used in the vaccine }\end{array}$ & [21] \\
\hline \multicolumn{5}{|l|}{ Tetanus } \\
\hline TT & $\begin{array}{l}\text { Plasmablasts } 6 \\
\text { days after three } \\
\text { consecutive } \\
\text { vaccinations, } \\
\text { separated by at } \\
\text { least } 1.5 \text { years }\end{array}$ & $\begin{array}{l}\text { Single-cell } \mathrm{V}_{\mathrm{H}}: \mathrm{V}_{\mathrm{L}} \\
\text { linkage PCR. DNA } \\
\text { cloning into } E \text {. coli and } \\
\text { Sanger sequencing of } \\
\text { TT-positive clones }\end{array}$ & $\begin{array}{l}\text { The number of somatic hypermutations was similar between individuals, and } \\
\text { did not increase through the study, indicating the limit had already been } \\
\text { reached through previous routine vaccinations. }\end{array}$ & [22] \\
\hline TT & $\begin{array}{l}\text { TT-specific } \\
\text { plasmablasts } 7 \\
\text { days, and TT- } \\
\text { specific memory B } \\
\text { cells } 9 \text { days after } \\
\text { vaccination }\end{array}$ & $\begin{array}{l}\text { Single-cell isotype- } \\
\text { specific } V_{\mathrm{H}} \text { and } \mathrm{V}_{\mathrm{L}} \mathrm{PCR} \\
\text { followed by Sanger } \\
\text { sequencing }\end{array}$ & $\begin{array}{l}\text { - VDJ segment usage, CDR3 length and distribution of somatic hypermutations } \\
\text { were similar among TT-specific plasmablasts and memory cells. }\end{array}$ & [23] \\
\hline \multicolumn{5}{|c|}{ Streptococcus pneumonia } \\
\hline $\begin{array}{l}\text { PS }(23 \\
\text { valent })\end{array}$ & $\begin{array}{l}\text { IgG plasmablasts } 7 \\
\text { days after } \\
\text { vaccination }\end{array}$ & $\begin{array}{l}\text { Single-cell } \mathrm{V}_{\mathrm{H}} \text { and } \mathrm{V}_{\mathrm{L}} \\
\text { PCR followed by } \\
\text { Sanger sequencing }\end{array}$ & $\begin{array}{l}\text { Cloned } 137 \text { mAbs against } 19 \text { of the } 23 \text { vaccine serotypes from } 4 \text { individuals. } \\
\text { Most antibodies were serotype-specific, but } 12 \% \text { cross-reacted with } 2 \text { or more } \\
\text { serotypes. }\end{array}$ & [77] \\
\hline $\begin{array}{l}\text { PS }(23 \\
\text { valent })\end{array}$ & $\begin{array}{l}\text { PPS4 or PPS } 14 \\
\text { specific B cells } 6 \\
\text { weeks after } \\
\text { vaccination }\end{array}$ & $\begin{array}{l}\text { Single-cell culture } \\
\text { followed by } \mathrm{V}_{\mathrm{H}} \text { PCR } \\
\text { and Sanger sequencing } \\
\text { of pooled, cultured } \\
\text { cells }\end{array}$ & $\begin{array}{l}\text { Analyzed over } 1300 \text { sequences from } 40 \text { individuals. Showed significant } \\
\text { differences in the antibody repertoire in young and elderly participants. } \\
\text { Elderly participants had a more clonal repertoire with less somatic } \\
\text { hypermutations. }\end{array}$ & [97] \\
\hline \multicolumn{5}{|c|}{ Haemophilus influenza B } \\
\hline $\begin{array}{l}\text { PS or PS- } \\
\text { DT or OC- } \\
\text { CRM }\end{array}$ & $\begin{array}{l}\text { Lymphocytes } 7 \\
\text { days after } \\
\text { vaccination }\end{array}$ & $\begin{array}{l}\text { Fusion of lymphocytes } \\
\text { to mouse myeloma } \\
\text { cells followed by } \\
\text { culture, } \mathrm{V}_{\mathrm{H}} \text { and } \mathrm{V}_{\mathrm{L}} \mathrm{PCR} \\
\text { and Sanger sequencing }\end{array}$ & $\begin{array}{l}\text { - Sequenced } 15 \text { cell lines from } 10 \text { individuals, secreting antibody against Hib PS. } \\
\text { Showed that these mAbs had undergone somatic hypermutation and } \\
\text { demonstrated clonality (all usingV } V_{\mathrm{H}} 3 \text { ) of the antibody repertoire after } \\
\text { vaccination. }\end{array}$ & [24] \\
\hline PS-DT & $\begin{array}{l}\text { Lymphocytes } 7 \\
\text { days after } \\
\text { vaccination }\end{array}$ & $\begin{array}{l}\text { Fusion of lymphocytes } \\
\text { to mouse myeloma } \\
\text { cells followed by } \\
\text { culture, } \mathrm{V}_{\mathrm{H}} \text { and } \mathrm{V}_{\mathrm{L}} \mathrm{PCR} \\
\text { and Sanger sequencing }\end{array}$ & $\begin{array}{l}\text { Sequenced } 4 \text { cell lines from } 4 \text { individuals, secreting antibody against Hib PS. } \\
\text { All used } V_{н} 3 \text {, but were composed of two different configurations of D and J } \\
\text { segment, and } V_{\mathrm{k}} \text { genes, indicating that the } 4 \text { cell lines were from } 2 \text { different } \\
\text { lineages. }\end{array}$ & [61] \\
\hline $\begin{array}{l}\text { PS or PS- } \\
\text { DT }\end{array}$ & $\begin{array}{l}\text { Lymphocytes } 7 \\
\text { days after } \\
\text { vaccination }\end{array}$ & $\begin{array}{l}\text { Fusion of lymphocytes } \\
\text { to mouse myeloma } \\
\text { cells followed by } \\
\text { culture, } V_{H} \text { PCR and } \\
\text { Sanger sequencing }\end{array}$ & $\begin{array}{l}\text { - Sequenced } 5 \text { cell lines from } 4 \text { individuals, secreting antibody against Hib PS. } \\
\text { Demonstrated that they all used } V_{\mathrm{H}} 3 \text {, but different } \mathrm{D} \text { and J segments. }\end{array}$ & [55] \\
\hline \multicolumn{5}{|c|}{ Publications where the antibody repertoire has been investigated using a high-resolution approach and next-generation sequencing } \\
\hline \multicolumn{5}{|l|}{ Influenza } \\
\hline TIV & $\begin{array}{l}\text { Memory B cells } 14 \\
\text { days after } \\
\text { vaccination (one } \\
\text { participant) }\end{array}$ & $\begin{array}{l}\text { High-throughput } \\
\text { single-cell } \mathrm{V}_{\mathrm{H}}: \mathrm{V}_{\mathrm{L}} \\
\text { linkage PCR and } 2 \times 250 \\
\text { bp Illumina sequencing }\end{array}$ & $\begin{array}{l}\text { - Validated accuracy of } \mathrm{V}_{\mathrm{H}}: \mathrm{V}_{\mathrm{L}} \text { pairings identified using their high-throughput } \\
\text { method. } \\
\text { - Identified } 240 \text { putatively influenza-specific CDR-H3:CDR-L3 pairings. }\end{array}$ & [18] \\
\hline $\begin{array}{l}\text { TIV or } \\
\text { LAIV }\end{array}$ & $\begin{array}{l}\text { PBMCs on the day } \\
\text { of vaccination, and } \\
\text { on day } 7 \text { and } 28 \\
\text { after two } \\
\text { vaccinations given } \\
\text { a year apart ( } 28\end{array}$ & $\begin{array}{l}\mathrm{V}_{\mathrm{H}} \text {-specific reverse } \\
\text { transcription and } 2^{\text {nd }} \\
\text { strand synthesis, with } \\
\text { the incorporation of } \\
\text { random nucleotide } \\
\text { tags, followed by PCR. }\end{array}$ & $\begin{array}{l}\text { - Showed different repertoire dynamics after TIV and LAIV vaccination. TIV } \\
\text { induced a stronger response, with more abundant IgG lineages than LAIV. } \\
\text { - Found shared antibody sequences on day } 7 \text { after two TIV vaccinations. } \\
\text { Hypothesized that these lineages are present after the second vaccination due } \\
\text { to memory B cell recall. Suggested that this method could be used to identify }\end{array}$ & [4] \\
\hline
\end{tabular}




\begin{tabular}{|c|c|c|c|c|}
\hline & participants) & $\begin{array}{l}\text { Custom } 100 \times 120 \mathrm{bp} \\
\text { Illumina sequencing } \\
\text { protocol, and } \\
\text { clustering of sequences } \\
\text { based on tags to } \\
\text { account for PCR bias } \\
\text { and error }\end{array}$ & cross-specific antibodies. & \\
\hline $\begin{array}{l}\text { TIV or } \\
\text { LAIV }\end{array}$ & $\begin{array}{l}\text { Naïve B cells, and } \\
\text { plasmablasts, on } \\
\text { the day of } \\
\text { vaccination and on } \\
\text { days } 7 \text { or } 8 \text { and } \\
\text { day } 28 \text { after } \\
\text { vaccination (17 } \\
\text { participants, three } \\
\text { age groups) }\end{array}$ & $\begin{array}{l}\mathrm{V}_{\mathrm{H}} \text {-specific multiplex } \\
\text { PCR, and Roche } 454 \\
\text { sequencing }\end{array}$ & $\begin{array}{l}\text { The influenza-specific antibody repertoire in older individuals was more } \\
\text { clonal, and had a greater mutational load than the repertoire in younger } \\
\text { individuals. } \\
\text { - In twins, the mutational load of the IgM repertoire was similar, but diverged } \\
\text { for the IgG repertoire, indicating that the naïve repertoire is more influenced } \\
\text { by individual genetics, but the memory repertoire is more influenced by } \\
\text { environmental stimuli. }\end{array}$ & [16] \\
\hline TIV & $\begin{array}{l}\text { PBMC's on } 18 \\
\text { timepoints around } \\
\text { two vaccinations } \\
\text { given a year apart } \\
\text { (one participant), } \\
\text { or } 10 \text { timepoints } \\
\text { around one } \\
\text { vaccination (two } \\
\text { participants). }\end{array}$ & $\begin{array}{l}\mathrm{V}_{\mathrm{H}} \text {-specific multiplex } \\
\text { PCR, and Roche } 454 \\
\text { sequencing }\end{array}$ & $\begin{array}{l}\text { - V and J segment usage differs between individuals, and is conserved within } \\
\text { individuals over time. } \\
\text { - Clustering sequences into clonal groups based on CDR3 identity showed clonal } \\
\text { expansion and contraction in response to the vaccine with different } \\
\text { participants exhibiting different dynamics. } \\
\text { - There are a small number of highly mutated, persistent clones found within all } \\
\text { individuals, potentially corresponding to long-lived B cell memory or } \\
\text { indicative of chronic infection. }\end{array}$ & [98] \\
\hline \multicolumn{5}{|c|}{ Tetanus } \\
\hline TT & $\begin{array}{l}\text { Plasmablasts } 7 \\
\text { days after } \\
\text { vaccination (one } \\
\text { participant) }\end{array}$ & $\begin{array}{l}\text { High-throughput } \\
\text { single-cell } V_{\mathrm{H}}: \mathrm{V}_{\mathrm{L}} \\
\text { linkage PCR and } 2 \times 250 \\
\text { bp Illumina sequencing }\end{array}$ & $\begin{array}{l}\text { Identified } 86 \text { putatively TT-specific CDR-H3:CDR-L3 pairings. Cloning ten of } \\
\text { these into HEK293K cells followed by competitive ELISA of the antibodies } \\
\text { produced showed them to be TT-specific. }\end{array}$ & [18] \\
\hline TT & $\begin{array}{l}\text { Bulk plasmablasts, } \\
\text { memory B cells, } \\
\text { and antigen- } \\
\text { specific } \\
\text { plasmablasts } 7 \\
\text { days and } 3 \text { months } \\
\text { after vaccination } \\
\text { (two participants) }\end{array}$ & $\begin{array}{l}\mathrm{V}_{\mathrm{H}} \text { and } \mathrm{V}_{\mathrm{L}} \text {-specific } \\
\text { multiplex PCR, and } \\
\text { Roche } 454 \text { sequencing. } \\
\text { Half the day } 7 \\
\text { plasmablasts were also } \\
\text { used for high- } \\
\text { throughput single-cell } \\
\mathrm{V}_{\mathrm{H}}: \mathrm{V}_{\mathrm{L}} \text { linkage PCR. Also } \\
\text { conducted proteomic } \\
\text { analysis of TT-specific } \\
\text { serum antibodies }\end{array}$ & $\begin{array}{l}\text { - Analyzed the serum antibody repertoire by using the } V_{\text {H }} \text { sequence database to } \\
\text { interpret results from high-resolution liquid chromatography tandom mass } \\
\text { spectrometry of the serum antibodies. } \\
\text { - Showed that } \sim 5 \% \text { of the plasmablast clonotypes identified by sequencing at } \\
\text { day } 7 \text { could subsequently also be detected in the serological response } 9 \\
\text { months after vaccination }\end{array}$ & [68] \\
\hline \multicolumn{5}{|c|}{ Co-administered } \\
\hline $\begin{array}{l}\text { TIV \& } \\
\text { PPV23 }\end{array}$ & $\begin{array}{l}\text { PBMCs on the day } \\
\text { of vaccination and } \\
\text { on day } 7 \text { and } 28 \\
\text { after vaccination } \\
\text { (14 participants, } \\
\text { two age groups) }\end{array}$ & $\begin{array}{l}\text { Semi-nested isotype } \\
\text { and } V_{\mathrm{H}-\text {-specific }} \\
\text { multiplex PCR, and } \\
\text { Roche } 454 \text { sequencing }\end{array}$ & $\begin{array}{l}\text { - The repertoire changed at day } 7 \text { post-vaccination, but returns to a baseline- } \\
\text { like state after } 28 \text { days. } \\
\text { - Comparing the repertoire in young and elderly individuals after vaccination } \\
\text { indicated clonal expansion is delayed in older individuals. Showed age-related } \\
\text { differences in IgA and IgM repertoire dynamics. }\end{array}$ & [13] \\
\hline
\end{tabular}

Abbreviations: TT, tetanus toxoid; PS, polysaccharide; OC, oligosaccharide; DT, diphtheria toxoid; TIV, trivalent inactivated influenza vaccine; LAIV, live attenuated influenza vaccine; PPV23, 23-valent pneumococcal polysaccharide vaccine. 